\title{
Herzklappenvitien
}

\section{Wann ist der richtige Operationszeitpunkt?}

\begin{abstract}
Nicht zu früh und nicht zu spät! So lautet die allgemeine Empfehlung für die Operation eines hämodynamisch wirksamen Vitium cordis. Doch was bedeutet dies für den richtigen Operationszeitpunkt beim einzelnen Patienten?
\end{abstract}

Der häufigste Klappenfehler in der heutigen Zeit ist die Aortenstenose.,,Unbestritten verschlechtert sich die Prognose betroffener Patienten bei diesem Vitium, sobald Symptome auftreten", sagte PD Dr. Alain Bernheim vom Stadtspital Triemli in Zürich. Ob eine asymptomatische schwere Aortenstenose operiert werden sollte müsse im Einzelfall unter Berücksichtigung der Prognose betroffener Patienten beantwortet werden.

\section{Indikatoren für eine schlechte Prognose} Wichtiger Prädiktor für ein schlechtes Outcome ist die Einschränkung der linksventrikulären Pumpfunktion; sie ist Ausdruck eines hämodynamisch fortgeschrittenen Stadiums einer schweren Aortenstenose. Aber auch bei asymptomatischen Patienten muss eine Einschränkung der linksventrikulären Pumpfunktion als unabhängiger Risikofaktor für kardiale Ereignisse angesehen werden.

Auch eine ausgeprägte Kalzifizierung und eine rasche Progression der Stenose (Zunahme der Jet-Velocity $>0,3 \mathrm{~m} / \mathrm{s} / \mathrm{Jahr}$ ) signalisieren eine ungünstige Prognose. Mit der Dobutamin-Stress-Echokardiografie kann eine höhergradige Aortenstenose bei linksventrikulärer Dysfunktion mit low output genauer differenziert werden. Kommt es unter Dobutamin zu einer proportionalen Zunahme des Cardiac output und des Klappengradienten ohne Änderung der Klappenöffnungsfläche, so spricht dies für eine höhergradige anatomische Stenose. Dagegen dürfte bei einer überproportionalen Zunahme des Cardiac output im Hinblick auf den Klappengradienten bei Zunahme der Klappenöffnungsfläche eine funktionelle Stenose im Sinne einer Pseudoobstruktion vorliegen. Darüber hinaus gibt die DubutaminStress-Echokardiografie auch wichtige Hinweise bezüglich der kontraktilen Reserve. „Eine Zunahme des Cardiac output um mehr als 20\% unter Dubutamin signalisiert eine

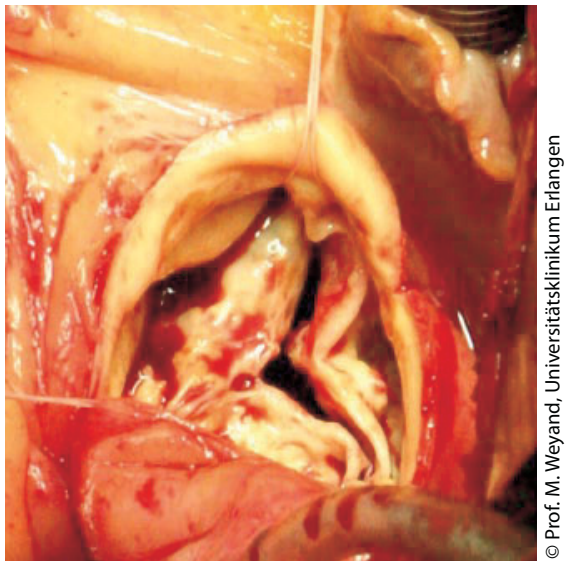

Bei schwerer Aortenstenose besteht immer die Indikation für den Klappenersatz.

günstige Prognose nach Aortenklappenersatz", so Bernheim.

\section{Algorithmus bei schwerer Aortenstenose} Bei Patienten mit Indikation für eine Bypassoperation sollte bereits eine mittelschwere Aortenstenose operativ korrigiert werden. Bei einer schweren symptomatischen Aortenstenose mit einer Klappenöffnungsfläche $<1$ $\mathrm{cm}^{2}$ besteht bei operablen Patienten immer die Indikation für den prothetischen Klappenersatz. Gleiches gilt für asymptomatische Patienten mit einer Auswurffraktion < 50\% und für Patienten mit einer stark verkalkten Aortenklappe und einer raschen Progression der Aortenstenose (Zunahme des Jet-Velocity $>0,3 \mathrm{~m} / \mathrm{s} / \mathrm{Jahr}$ ). Sollten diese Kriterien nicht zutreffen, empfiehlt sich eine Belastungsuntersuchung. Ist diese pathologisch bzw. treten unter Belastung Symptome auf, sollte ebenfalls die Indikation für den Klappenersatz großzügig gestellt werden. Ansonsten sollte die Untersuchung in Abständen von 6-12 Monaten wiederholt werden.

\section{Risikofaktoren bei Aorteninsuffizienz: Dilatation und LV- Dysfunktion}

Bei der chronischen Aorteninsuffizienz kommt es zu einer exzentrischen Hypertrophiedes linken Ventrikels infolge chronischer Volumenüberlastung. Dies führt zu einer Erhöhung von pre- und afterload. In kompensiertem Zustand kann trotz afterloadErhöhung das Schlagvolumen aufrecht er- halten werden. „Nach einem Klappenersatz sinkt das afterload, was für die Erholung des Ventrikels günstig ist", so Bernheim.

Ebenso wie bei der Aortenstenose stellt auch bei der Aorteninsuffizienz das Auftreten von Symptomen eine Operationsindikation dar. Bei asymptomtischen Patienten mit Aorteninsuffizienz sollte eine Operationsindikation dann gestellt werden, wenn die Auswurffraktion $<50 \%$ beträgt oder der enddiastolische Durchmesser $>70 \mathrm{~mm}$ oder der endsystolische Durchmesser $>50 \mathrm{~mm}$ beträgt.

\section{Schwere Mitralinsuffizienz früher operieren}

Die Mitralinsuffizienz verursacht eine reine Volumenbelastung, wobei in chronisch kompensiertem Zustand das afterload normal ist. Postoperativ kommt es zu einem Anstieg des afterload, was für einen kranken Ventrikel problematisch sein kann. „Deshalb sollte nicht zu lange mit der Operation gewartet werden", so Bernheim. Bei asymptomatischen Patienten sollten wie bei der Aortensklerose Risikofaktoren berücksichtigt werden. Dazu gehörten neben Symptomen auch Alter, Vorhofflimmern, linksatriale Dilatation, linksventrikuläre Dilatation, verminderte Auswurffraktion und Ausmaß der Mitralinsuffizienz. Konkret sollte heute bei asymptomatischen Patienten mit einer schweren organischen Mitralinsuffizienz die Operationsindikation bei einer Auswurffraktion > $60 \%$ dann gestellt werden, wenn der endsystolische Durchmesser $>45 \mathrm{~mm}$ beträgt oder Vorhofflimmern oder eine pulmonal-arterielle Hypertonie mit einem systolischen Pulmonalarteriendruck von $>50 \mathrm{mmHg}$ vorliegt.

Bei Patienten mit einer schweren funktionellen Mitralinsuffizienz als Folge einer linksventrikulären Geometriestörung ist die Datenlage bezüglich Operationserfolg sehr limitiert. „Die wenigen vorhandenen Daten implizieren, dass eine Mitralklappenoperation erwogen werden kann, falls der Patient ansonsten therapierefraktär ist und kaum Komorbiditäten aufweist", so Bernheim.

Dr. med. Peter Stiefelhagen II

II Quelle: medArt Basel '11, 20.-24. Juni 2011, Basel 